\title{
Serum Vitamin D levels in pediatric patients and its association with COVID-19 clinical manifestation: A meta-analysis and systematic review
}

\author{
Andrew Jonatan $1{ }^{*}{ }^{*}$ and Retno Asih Setyoningrum ${ }^{2,3}$ \\ ${ }^{1}$ Indrapura Field Hospital, Surabaya, Indonesia ATTN: Andrew Jonatan Indrapura ST No. 17., Surabaya, Indonesia. \\ 2 Departement of Pediatrics, Faculty of Medicine, Airlangga University, Surabaya, Indonesia. \\ ${ }^{3}$ dr. Soetomo General Hospital, Surabaya, Indonesia.
}

GSC Biological and Pharmaceutical Sciences, 2021, 17(02), 076-085

Publication history: Received on 30 September 2021; revised on 12 November 2021; accepted on 14 November 2021

Article DOI: https://doi.org/10.30574/gscbps.2021.17.2.0328

\begin{abstract}
Background: Vitamin D is involved in human immune system homeostasis and thought to be beneficial for COVID-19 patients, including pediatric population. However, there is still a paucity of information on association of serum vitamin D levels and COVID-19 clinical manifestation in pediatric patients. This study evaluated the association between serum vitamin D levels and clinical manifestations of COVID-19 in pediatric patients.
\end{abstract}

Methods: We searched PubMed and Google Scholar for articles reporting association between vitamin D and COVID-19 clinical manifestations in pediatric patients. We searched for English-written articles only. PRISMA-MOOSE guideline and quality checklist was complied and statistical analysis was performed using RevMan 5.4.

Results: Ten studies with total of 1,225 patients reported were reviewed. Low vitamin D levels was significantly associated with higher incidence of severe COVID-19 with pooled OR was 5.57 (1.59-19.55; $\mathrm{p}=0.007)$. Clinical symptoms were compared between low and normal vitamin D levels. Incidence of fever, cough, ageusia, anosmia, headache, and fatigue were not significantly different between group, with pooled RR was $2.13(0.13-33.73 ; \mathrm{p}=0.59), 1.25(0.25-6.22$; $\mathrm{p}=0.78), 0.47$ (0.05-4.76; p=0.52), 1.25 (0.21-7.41; $\mathrm{p}=0.81), 0.91$ (0.33-2.55; p=0.86), and 1.02 (0.47-2.22; p=0.96), respectively. The mean count of leukocytes and lymphocytes in the two groups were also not significantly different with pooled RR was $-0.49(-1.39-0.41 ; \mathrm{p}=0.28)$, and $-0.73(-1.65-0.20 ; \mathrm{p}=0.12)$, respectively.

Conclusion: Low serum vitamin D level $(<20 \mathrm{ng} / \mathrm{ml})$ is associated with the severity of COVID-19 in pediatric patients, but do not affect symptoms and laboratory parameters. Vitamin D supplementation might be beneficial for children undergo medical quarantine and isolation.

Keywords: Children; COVID-19; Pediatric Patients; Vitamin D

\section{Introduction}

Severe acute respiratory syndrome coronavirus 2 (SARS-CoV-2) first appeared in December 2019 and was declared a global pandemic in March 2020[1]. SARS-CoV-2 is a virus that binds to the angiotensinconverting enzyme 2 (ACE2) as its receptor, which is widely expressed in human tissues, including pulmonary epithelium, myocardium, and vascular endothelium, which are responsible for the clinical manifestations of coronavirus disease 2019 (COVID-19)[2]. COVID19 is a syndrome with many clinical manifestations ranging from flu-like symptoms to severe complications that require treatment in intensive care unit (ICU) for all age groups[3]. As World Health Organization (WHO) declares COVID-19 as a pandemic, most countries are implementing several policies to reduce the spread of SARS-CoV-2, such as social

\footnotetext{
* Corresponding author: Andrew Jonatan

Indrapura Field Hospital, Surabaya, Indonesia ATTN: Andrew Jonatan, MD. Indrapura ST No. 17., Surabaya, Indonesia. 
distancing, quarantine, isolation, and travel restrictions. Despite the importance of spread control measurement during the COVID-19 pandemic, prolonged pandemic-related home confinement negatively impacts children's health, especially the growth of children[4]. Previous studies have shown that children that stayed indoor for long periods tend to be more physically inactive, adopt an unhealthy diet, and have limited sun exposure -putting children at risk for vitamin D deficiency[5,6].

Vitamin D is a steroid hormone that plays a vital role in maintaining calcium homeostasis and bone health, as well as boosting the immune system by modulating immune regulation[7-9]. Vitamin D receptors are present in various cells involved in immune regulation, such as monocytes, $\mathrm{T}$ and B lymphocytes, and dendritic cells. Therefore, infants and children are recommended to have a vitamin D intake of at least 400 IU per day[10]. Sources of vitamin D can be obtained through food intake or supplements as much as $10 \%$, and the other $90 \%$ should be obtained from exposure to sunlight[11]. However, vitamin D intake is also determined by environmental exposure and endogenous factors such as skin pigmentation, sun protection, exposure to ultraviolet-B (UVB) radiation, and the part of the skin that covered in clothes[8,12]. Thus, vitamin D levels could vary significantly between individuals. Previous research has shown that regular sun exposure is sufficient to meet the needs of vitamin $\mathrm{D}[6,13]$. Considering the importance of the role of vitamin D, especially in children, implementing policies to reduce the spread of COVID-19 by advising the increase of vitamin D supplementation should be concerned. Previous studies have shown that vitamin D deficiency in both adults and children increases the susceptibility and severity of COVID-19[14,15]. Vitamin D supplementation has been shown to reduce the risk of severe clinical manifestation and mortality in COVID-19[14,16]. Therefore, we conducted a metaanalysis and systematic review to demonstrate a possible association between vitamin D levels and COVID-19 infection in pediatric patients.

\section{Methods}

This study was prepared according to the recommendations of the Preferred Reporting Items for Systematic Review and Meta-Analysis (PRISMA)-MOOSE guidelines. Before conducting the research, the analysis method, inclusion, and exclusion criteria have been determined.

\subsection{Literature Search, Systematic Review, and Meta-Analysis}

We performed literature search using google scholar in all over the web and we also searched for relevant literature in PubMed database. Keywords used as query in google scholar were "Vitamin D", "COVID-19", and "Pediatric" and their synonyms. We searched for articles published in English between 2019 and 2021. PubMed database was also searched to identify articles examining the association between vitamin D levels and pediatric patient outcomes for COVID-19 using the following keywords "Vitamin D", "COVID-19", and "Pediatric".

For systematic review, we did not exclude relevant in-vitro, in-vivo, meta-analyses, literature reviews, and systematic reviews. Meanwhile, we performed meta-analysis based on articles found in PubMed. A total of 85 articles were found. In the end, we selected ten articles that were also relevant to the keywords that met the PICOS criteria in our study. The studies used in our meta-analysis were studies that met the following PICOS criteria: (1) Participants: pediatric patients (younger than 18 years old) with COVID-19, (2) Intervention: normal or high serum Vitamin D levels, (3) Comparison: low serum vitamin D, (4) Outcomes: patients' symptoms, laboratory findings, and severity of COVID-19, (5) Study Design: All studies comparing The Effect of Normal/High Vitamin D and Low Vitamin D status in COVID-19 Pediatric Population.

\subsection{Data Extraction}

The authors (A.J.) screened and abstracted each study to determine which articles met the inclusion criteria. Information extracted from each study included in the meta-analysis was as follows: first author name, year of publication, study design, country, sample size, age, vitamin D level in pediatric patients with COVID-19.

\subsection{Data Analysis}

All statistical analysis were performed using RevMan v.5.4 (The Cochrane Collaboration, Oxford, Copenhagen, Denmark). All tests were 2 -sided, and a p-value of less than 0.05 was considered statistically significant. The MantelHaenszel method was used to analyze the dichotomous outcome, and the results were reported in odds ratios (OD) with 95\% confidence interval (CI). An inverse variance model with 95\% CI was used to analyze the difference in continuous outcomes, and the data were reported as mean difference (MD). Results are presented in risk ratio (RR) with $95 \% \mathrm{CI}$ for the dichotomous measure. 


\section{Results}

\subsection{Characteristic of Study}

A detailed description of the study selection process is presented in Figure 1. We found 85 articles in the PubMed Database. Six articles were excluded because they were duplicates, and 60 articles were also excluded due to irrelevant on the title. Nine articles were excluded due to irrelevant based on the PICOS criteria in our study. Finally, ten articles were included in our study.

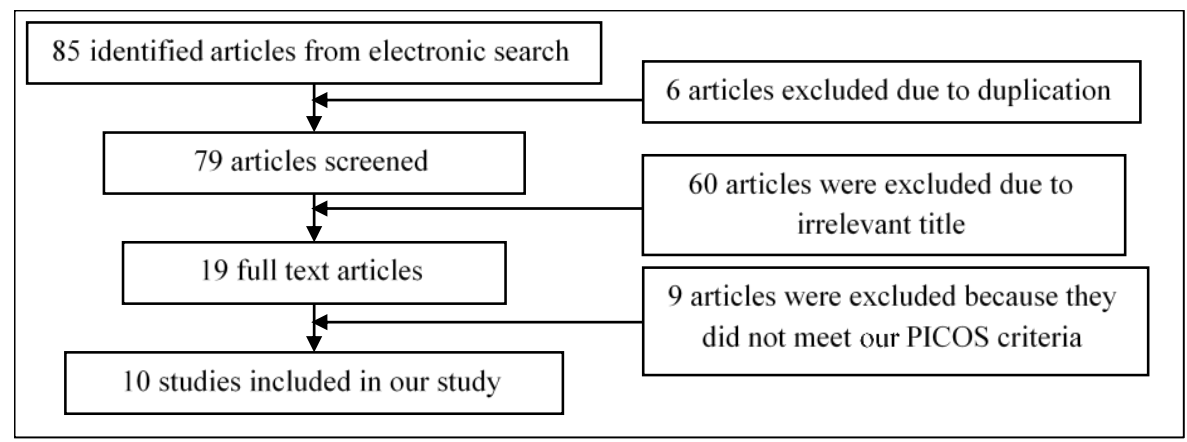

Figure 1 Flow diagram showing stages of the database search and study selection as PRISMA-MOOSE guideline

We studied evidence from ten articles with different study designs. It includes two reviews of published articles from Ireland and India, and the rest are either retrospective, cohorts, or cross-sectional. Two studies that assessed the role of vitamin D levels in disease severity are included in the meta-analysis to get the cumulative chance of developing poorer outcomes in case of vitamin D deficiency. Two studies that assessed the clinical symptoms and laboratories examination associated with vitamin D deficiency were also included in our meta-analysis.

Characteristic details of the included studies are presented in Table 1. Studies were conducted from various continents including, Europe, America, and Asia. The sample size of the population varied widely, ranged from 15 patients to 884 patients. One study included pediatric and adult populations, and the others presented data exclusively from patients aged younger and equal to 18 years old. Our evidence synthesis included studies published from December 2019 to June 2021.

\subsection{Meta-Analysis Results}

A pool analysis of all studies reporting the effect of vitamin D levels on severity, clinical symptoms, as well as lymphocyte and leukocyte counts in pediatric patients with COVID-19.

\subsection{Serum Vitamin D Levels and COVID-19 Severity}

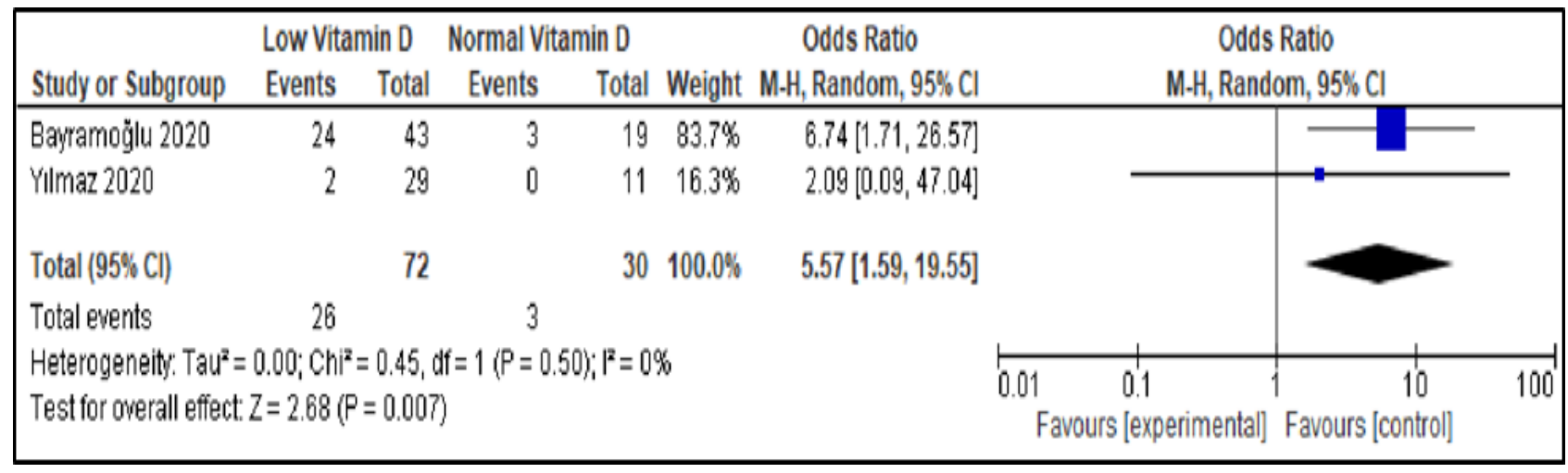

Figure 2 Forest plot of COVID-19 severity between the low and normal serum level of vitamin D. Low vitamin D favors severity of COVID-19 (Odds ratio (OD) 5.57; 95\% CI, 1.59-19.55; p=0.007)

Two studies provided vitamin D levels in pediatric COVID patients, and a pooled prevalence of vitamin D deficiency was analyzed. Meta-analysis of the quantitative data (two studies) showed a pooled Odd Ratio of vitamin D deficiency as 
5.57 (1.59-19.55; $\mathrm{p}=0.007$ ) (Figure 2). Some studies have also pointed to an inverse relationship between vitamin $\mathrm{D}$ levels and COVID-19 infection risk, indicating higher odds of infection in individuals with vitamin D deficiency than those with normal serum vitamin D levels.

\subsection{Serum Vitamin D Levels and COVID-19 Clinical Symptoms}

Clinical symptoms (fever, cough, ageusia, anosmia, headache, and fatigue) were compared between two studies with data of low vs normal serum vitamin D levels. Incidence of fever, cough, ageusia, anosmia, headache, and fatigue were not significantly different with pooled RR values were $2.13(0.13-33.73 ; p=0.59), 1.25(0.25-6.22 ; p=0.78), 0.47(0.05$ 4.76; $\mathrm{p}=0.52) .1 .25$ (0.21-7.41; $\mathrm{p}=0.81), 0.91(0.33-2.55 ; \mathrm{p}=0.86)$, and $1.02(0.47-2.22 ; \mathrm{p}=0.96)$ (Figure 3), respectively.

\subsection{Serum Vitamin D Levels and Laboratory Findings}

Laboratorium examination (leucocytes and lymphocytes count) was compared between studies with lower vitamin D and normal vitamin D levels. The average levels of leukocytes and lymphocytes in the two groups were also not significantly different with pooled RR $-0.49(-1.39-0.41 ; p=0.28),-0.73(-1.65-0.20 ; p=0.12)$ (Figure 4), respectively.

Table 1 Patient characteristics in included studies

\begin{tabular}{|c|c|c|c|c|c|c|c|}
\hline No. & $\begin{array}{l}\text { Author, Study } \\
\text { Year }\end{array}$ & Study Design & Study Period & Country & $\begin{array}{l}\text { Total COVID- } \\
19 \text { Patients }\end{array}$ & $\begin{array}{l}\text { Mean Age } \\
\text { (Population) }\end{array}$ & $\begin{array}{l}\text { Male } \\
(\%)\end{array}$ \\
\hline 1 & $\begin{array}{l}\text { Darren, } \\
2021[17]\end{array}$ & Cohort & $\begin{array}{l}\text { April } 12 \text { - June } \\
25,2020\end{array}$ & England & 18 & $8.9(0.3-14.6)$ & $55.56 \%$ \\
\hline 2 & $\begin{array}{l}\text { Molla, } \\
2021[18]\end{array}$ & Cross Sectional & $\begin{array}{l}\text { May } 15 \text { - June } \\
15,2020\end{array}$ & Turkey & 49 & $13(8-18)$ & $47 \%$ \\
\hline 3 & $\begin{array}{l}\text { Fekete, } \\
2021[19]\end{array}$ & $\begin{array}{l}\text { Literature } \\
\text { Review }\end{array}$ & $\begin{array}{l}\text { December } 2019 \\
\text { - } 30 \text { December } \\
2020\end{array}$ & Ireland & 7 Paper used & $(0-16)$ & - \\
\hline 4 & $\begin{array}{l}\text { Isoldi, } \\
2021[20]\end{array}$ & Prospective & $\begin{array}{l}\text { April } 6 \text { - June 5, } \\
2020\end{array}$ & Italy & 15 & $\begin{array}{c}\text { median } \\
12.2(4.8-7.8)\end{array}$ & $53.33 \%$ \\
\hline 5 & $\begin{array}{l}\text { Yilmaz, } \\
2020[21]\end{array}$ & Retrospective & $\begin{array}{l}\text { March - May, } \\
2020\end{array}$ & Turkey & 40 & $\begin{array}{c}8.48 \pm 2.32 \\
(3 \mathrm{~m}-18 \mathrm{y})\end{array}$ & $47.5 \%$ \\
\hline 6 & $\begin{array}{l}\text { Bayramoğlu, } \\
2021[22]\end{array}$ & Retrospective & $\begin{array}{l}\text { March - May, } \\
2020\end{array}$ & German & 103 & $\begin{array}{c}12.2 \pm 4.92(1- \\
17)\end{array}$ & $52.4 \%$ \\
\hline 7 & $\begin{array}{l}\text { Alpcan, } \\
2021[23]\end{array}$ & Retrospective & $\begin{array}{ll}\text { May } & 25- \\
\text { December } & 24, \\
2020 & \end{array}$ & Turkey & 75 & $\begin{array}{c}10.7 \pm 5.5(1- \\
18)\end{array}$ & $57.3 \%$ \\
\hline 8 & Söbü, 2021[24] & Retrospective & $\begin{array}{l}\text { March - April, } \\
2020\end{array}$ & Turkey & 30 & $\begin{array}{c}10.48 \pm 4.75 \\
(1.8- \\
17.6)\end{array}$ & $50 \%$ \\
\hline 9 & Katz, 2021[25] & Retrospective & $\begin{array}{l}\text { January - June } \\
2020\end{array}$ & America & 887 & $(0-64)$ & $56.44 \%$ \\
\hline 10 & Shah, 2021[14] & $\begin{array}{l}\text { Systematic } \\
\text { Review }\end{array}$ & $\begin{array}{l}\text { December } 2019 \\
\text { - June } 2021\end{array}$ & India & - & $(0-18)$ & - \\
\hline
\end{tabular}


GSC Biological and Pharmaceutical Sciences, 2021, 17(02), 076-085

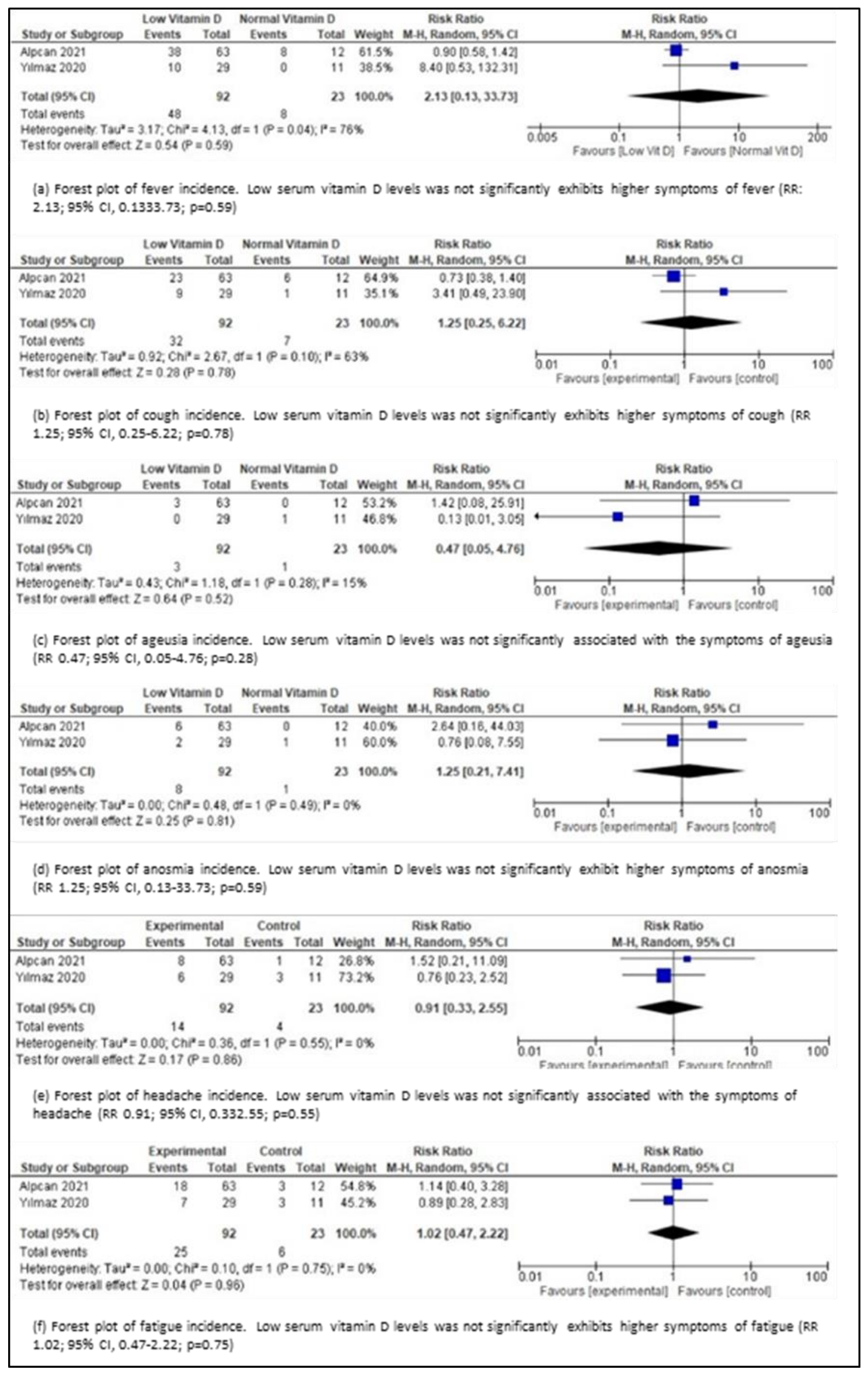

Figure 3 Forest Plots of symptomps incidence in COVID-19 pediatric patients 


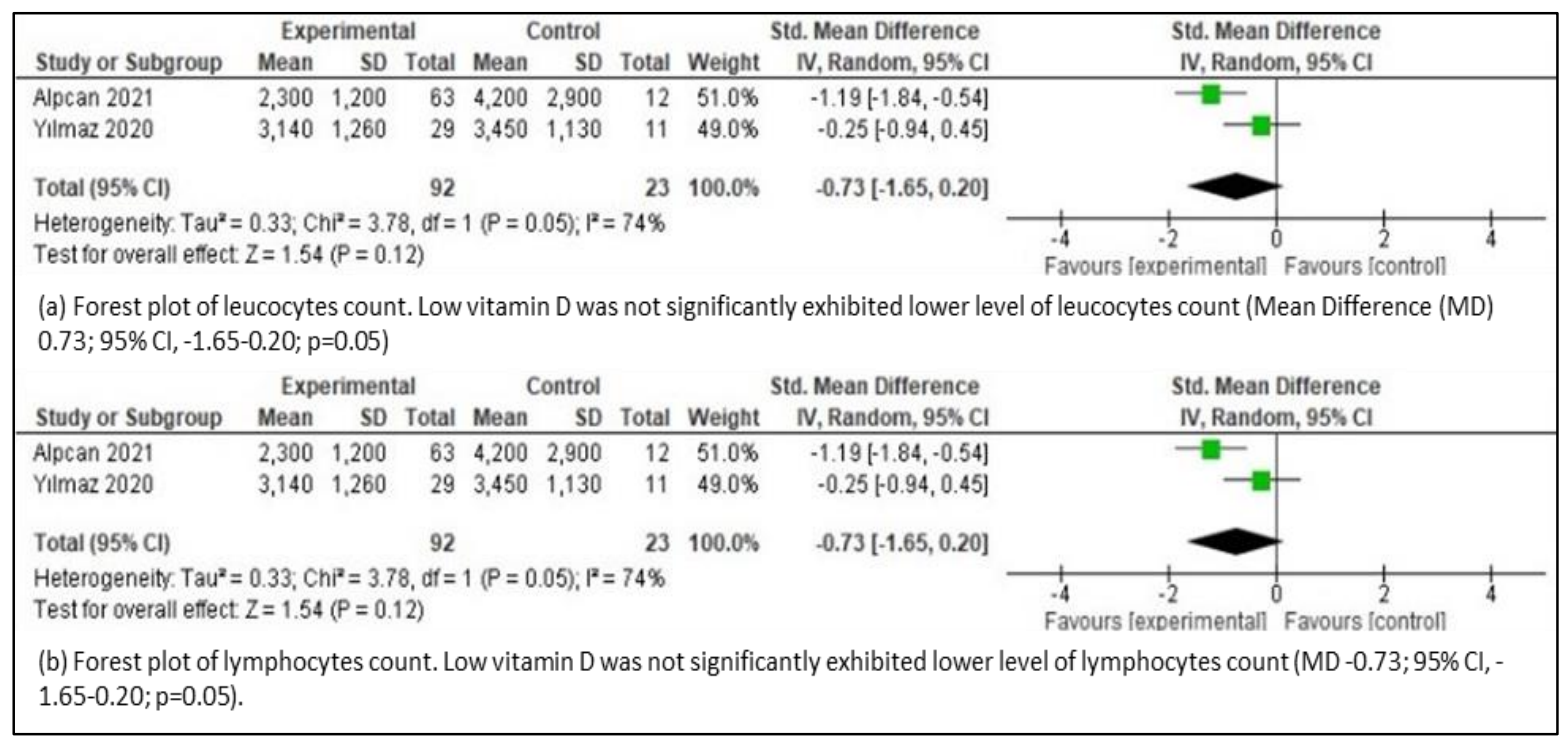

Figure 4 Forest plot of leucocyte and lymphocytes count

\section{Discussion}

The role of macro and micronutrients in modulating the immune system response has been a continous subject of research for long, resulting in many discoveries. Vitamin D is a micronutrient known to play a role in calcium metabolism and bone homeostasis. In addition, vitamin D regulates innate and adaptive immunity and further modulates the immune response to viral and bacterial infections[26,27]. A randomized controlled study showed that vitamin D supplementation significantly reduces the risk of acute respiratory tract infections[27]. Many reports are showing an association between vitamin D deficiency and the risk of COVID-19 infection. This report is supported by identification of Calcitriol (the active form of vitamin D) as a regulator of the Renin-Angiotensin System (RAS), whose overactivity is associated with a poor prognosis of COVID-19[28]. Significant associations between vitamin D, sun exposure, the prognosis of COVID-19 patients, and disease severity have also been reported in adults[15,29,30]. One systematic study of pediatric COVID-19 patients showed that half of the study population was deficient in vitamin D, and there is also an association between vitamin D deficiency with a greater risk of infection and a poorer prognosis in pediatric patients[14].

Vitamin D3 is produced from 7-dehydrocholesterol (7DHC) in the skin, an intermediate in cholesterol synthesis. Exposure to ultraviolet B (UVB) light in the wave length of 290-315 nm will produce previtamin D (PreD3). After PreD3 is formed, thermal isomerization converts PreD3 to vitamin D3 (Vit. D3)[31]. Then, vitamin D3 and vitamin D2 (a form of vitamin D originating from the gastrointestinal system) are carried by vitamin D-binding protein (VDBP), and chylomicrons to the liver, where it undergoes hydroxylation to form 25 xycholecalciferol [25(OH)D] with the help of the enzyme 25-hydroxylase, cytochrome p450 CYP2R1 and cytochrome p450 CYP27A1[32]. Most of the 25-hydroxylase enzymes are expressed in the liver. All are members of the cytochrome P450 family (CYP2C11, CYP2D25, CYP27A1, CYP3A4, CYP2R1, and CYP2J2/3)[32-34]. Among them, CYP2R1 and CYP27A1 are considered the most promising candidates in the process of vitamin D hydroxylation. The hydroxylation process continues, as 25(OH)D carried by VDBP to the kidney, where $1 \alpha$, hydroxylase, and CYP27B1 convert it to the active form: $1 \alpha, 25$ dihydroxycholecalciferol $[1,25(\mathrm{OH}) 2 \mathrm{D}$, or calcitriol]. Calcitriol then binds to the intracellular vitamin D receptor (VDR) in the target gene[35]. Further hydroxylation process by 24-hydroxylase and CYP24A1 inactivates 25(OH)D and 1,25(OH)2D and converts them to inactive forms: 24,25(OH)2D and 1,24,25(OH)3D (Figure 5) [35]. According to the American Endocrine Society, a serum 25(OH)D level below $20 \mathrm{ng} / \mathrm{mL}(50 \mathrm{nmol} / \mathrm{L})$ is defined as vitamin D deficiency, whereas a serum 25(OH)D level between $21-29 \mathrm{ng} / \mathrm{mL}(52.5-72.5 \mathrm{nmol} / \mathrm{L})$ is defined as vitamin D insufficiency[34]

Sunlight provide energi source to produce $90 \%$ of the vitamin D required in most people. The synthesis and endogenous metabolism of vitamin D cannot be carried out without sunlight. It has been described that the synthesis of endogenous vitamin D initiated through sun exposure produces pre-vitamin D3, which is converted to an active form and acts on target cells in bone and immune cells [35]. Activation of vitamin D receptors can increase the expression of genes related to both innate and adaptive immunity. The receptor was expressed in immune cells, including T cells (CD4+ and CD8+), $B$ cells, and antigen-presenting cells (macrophages and dendritic cells). Vitamin D can decrease pro-inflammatory cytokines by regulating macrophages and activity of T lymphocytes, such as expansion and migration of $\mathrm{T}$ cells in the 
lung during SARS-Cov-2 infection[36,37]. Vitamin D can up-regulate the regulatory type-2 (T-reg) cells and reduce expression of Th17-stimulating cytokines. Next, expression of pro-inflammatory type-1 cytokines such as IL-8, IL-12, IL-6, IL-17, and TNF- $\alpha$, can be reduced by 25-hydroxyvitamin D and 1,25(OH)2D[36,38]. On the other hand, vitamin D supplementation in COVID-19 pediatric patients can reduce the interaction of CD 26-R with SARS-COV-2 in lung cells and immune cells[36,39]. Modulation of CD26, also known as dipeptidyl peptidase 4 (DPP4), may prevent the progression of COVID-19 (Figure 6)[39]. Increased inflammation and vitamin D insufficiency are implicated as the cause of morbidity or mortality in COVID-19 patients.

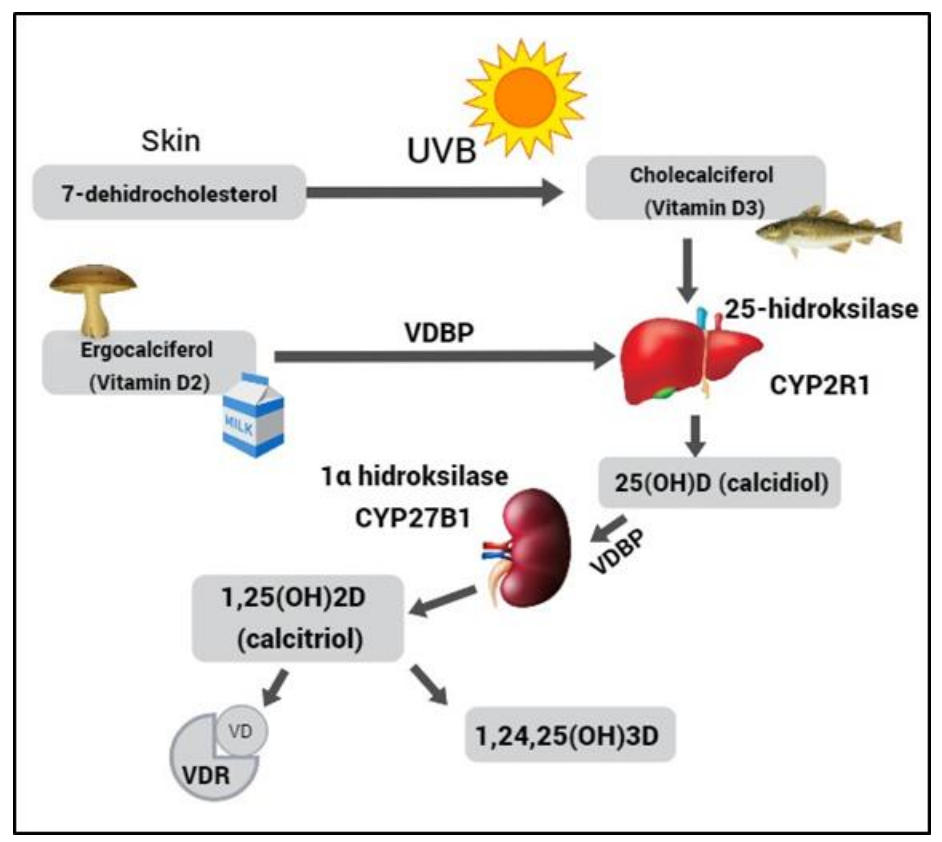

Figure 5 Vitamin D synthesis and metabolism. A sequential metabolic process converts the inactive vitamin D into an active form[35]

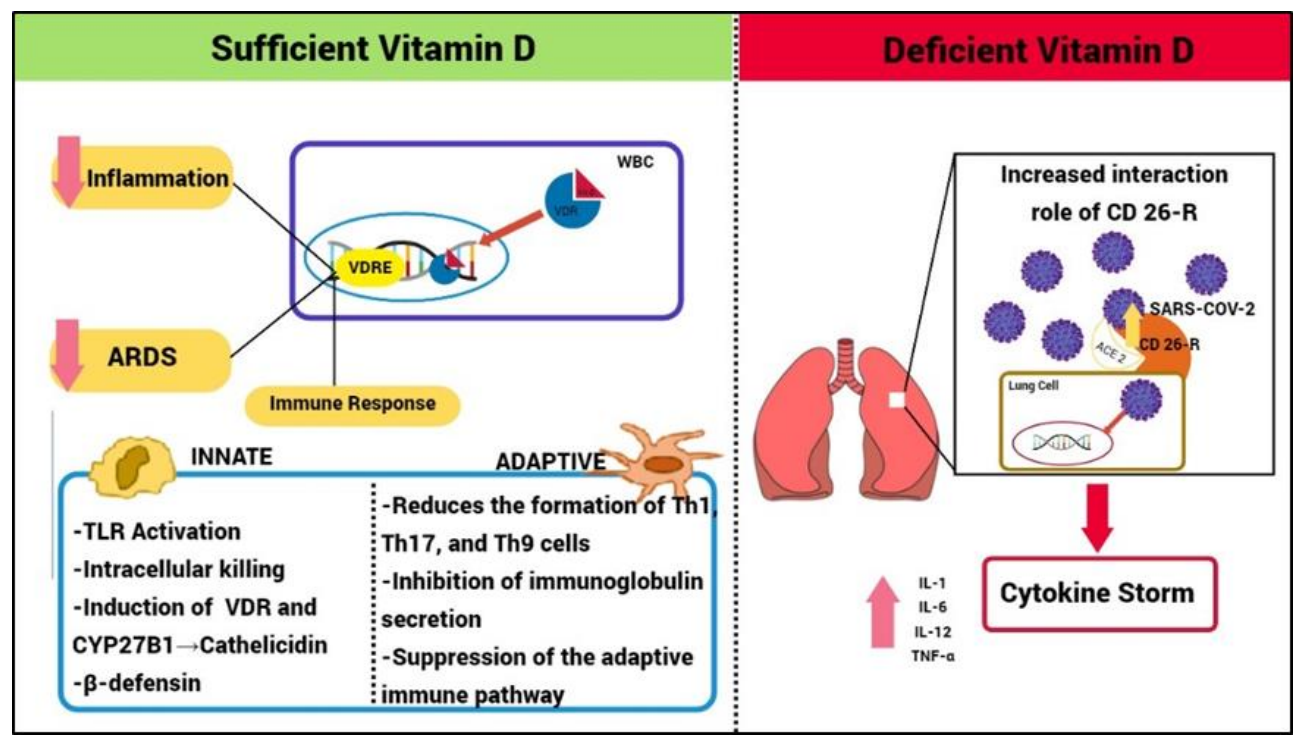

Figure 6 The proposed mechanisms of vitamin D in Covid-19[39]. On the left side, the principal functions of vitamin D. Especially anti-inflammatory properties with subsequent reduction of ARDS, and its immunomodulatory role on the innate and adaptive immune systems. The right side shows the "Cytokine Storm" caused by SARS-Cov-2 in a deficient vitamin D and the possible interaction role of DPP-4/CD 26R

Patients with infectious disease and vitamin D sufficient, promote regulation of inflammatory cytokine and antiviral responses. Sufficient Vitamin D levels also promote immunoprotective NK-T cells and preserve epithelial junction 
integrity and vascular permeability, thus minimizing pulmonary damage and ARDS[37]. Based on our findings in this study, the severity of COVID-19 increased in patients with low serum level of vitamin D.

A decrease in peripheral lymphocytes has been observed and associated with a severe clinical course of COVID-19. The proposed mechanism is functional depletion of ACE2 expressing and SARS-CoV2- infected lymphocytes in the early stages of the disease, which causes the impairment of viral immunity. It has also shown that the lymphocyte count reaches the lowest values while the inflammatory cytokine levels are the highest on days 4 to 6 . The latest study showed that the clinically severe group with significantly lower vitamin D levels and lymphocyte count had significantly higher inflammation markers[40,41]. Furthermore, the lower $25(\mathrm{OH}) \mathrm{D}$ level was associated with higher inflammation markers (CRP and fibrinogen) and a lower lymphocyte count. Therefore, vitamin D deficiency may have a role in hyperinflammation and low lymphocyte count in COVID-19[22] ${ }^{35}$. Accoriding to our study, low vitamin D level exhibits a lower level of lymphocyte count, although not significant. There is an increase of clinical symptoms number of COVID19 pediatric patients within the low vitamin D group, such as fever, cough, anosmia, and fatigue, but it is not significantly associated. At the same time, there is no correlation between ageusia and headache in the low-level vitamin $\mathrm{D}$ group.

\section{Conclusion}

Our meta-analysis showed that low serum vitamin D levels significantly affect the severity of COVID-19 pediatric patients. Low serum vitamin D levels have a weak correlation with the incidence of fever, cough, anosmia, and fatigue. Meanwhile, there is no correlation between the incidence of ageusia and headache with the low level of serum vitamin D. Low serum vitamin D levels are not significantly associated with lower leucocytes and lymphocytes count in COVID19 pediatric patients. It is essential to mantain sufficient serum vitamin D levels, thus supplementation of vitamin D, especially in deficienct and risk groups are indicated.

\section{Compliance with ethical standards}

\section{Acknowledgments}

The authors would like to thank all those who have helped the authors in this research.

\section{Disclosure of conflict of interest}

The authors declare that there is no conflict of interest in this study.

\section{References}

[1] Wang F, Lancet CZ.-T. U. What to do next to control the 2019-nCoV epidemic? thelancet.com. 2020.

[2] Mosleh W, Chen K, Pfau SE, Vashist A. Endotheliitis and endothelial dysfunction in patients with COVID-19: its role in thrombosis and adverse outcomes. 2020.

[3] Amdal, C. D. et al. Health-related quality of life issues, including symptoms, in patients with active COVID-19 or post COVID-19; a systematic literature review. Qual. Life Res. 2021; 1-15.

[4] Wang G, Zhang Y, Zhao J, Zhang J, Jiang F. Mitigate the effects of home confinement on children during the COVID19 outbreak. Lancet. 2020; 395: 945-947.

[5] Nowson C. A. et al. Vitamin D and health in adults in Australia and New Zealand: a position statement. Med. J. Aust. 2012; 196: 686-687.

[6] Yu L. et al. Effect of pandemic-related confinement on vitamin D status among children aged 0--6 years in Guangzhou, China: a cross-sectional study. Risk Manag. Healthc. Policy. 2020; 13: 2669.

[7] Norman AW. From vitamin D to hormone D: fundamentals of the vitamin D endocrine system essential for good health. Am. J. Clin. Nutr. 2008; 88: 491S--499S.

[8] Lips P. Vitamin D physiology. Prog. Biophys. Mol. Biol. 2006; 92: 4-8.

[9] Wang Z. et al. Association of vitamin D deficiency with COVID-19 infection severity: Systematic review and metaanalysis. Clin. Endocrinol. (Oxf). 2021. 
[10] Yousfi, N., Bragazzi, N. L., Briki, W., Zmijewski, P. \& Chamari, K. The COVID-19 pandemic: how to maintain a healthy immune system during the lockdown--a multidisciplinary approach with special focus on athletes. Biol. Sport. 2020; 37: 211.

[11] Rustecka, A. et al. The Impact of COVID-19 Pandemic during 2020--2021 on the Vitamin D Serum Levels in the Paediatric Population in Warsaw, Poland. Nutrients. 2021; 13: 1990.

[12] Wagner CL, Greer FR, others. Prevention of rickets and vitamin D deficiency in infants, children, and adolescents. Pediatrics. 2008; 122: 1142-1152.

[13] Nowson CA, Margerison C. Vitamin D intake and vitamin D status of Australians. Med. J. Aust. 2002; 177: 149152.

[14] Shah K, Varna VP, Pandya A, Saxena D. Low vitamin D levels and prognosis in a COVID-19 pediatric population: a systematic review. QJM An Int. J. Med. 2021.

[15] Weir EK, Thenappan T, Bhargava M, Chen Y, Does vitamin D deficiency increase the severity of COVID-19? Clin. Med. (Northfield. Il). 2020; 20: e107.

[16] Shah K, Saxena D, Mavalankar D. Vitamin D supplementation, COVID-19 and disease severity: a meta-analysis. QJM An Int. J. Med. 2021; 114: 175-181.

[17] Darren A. et al. Vitamin D status of children with Paediatric Inflammatory Multisystem Syndrome Temporally associated with Severe acute respiratory syndrome coronavirus 2 (PIMS-TS). Br. J. Nutr. 2021; 1-26.

[18] Molla GK, Uzun ÖÜ, Koç N, Ye \csil, B. Ö. \& Bayhan, G. \. I. Evaluation of nutritional status in pediatric patients diagnosed with Covid-19 infection. Clin. Nutr. ESPEN. 2021.

[19] Fekete T. In adults and children in the ED with COVID-19 symptoms or a confirmed contact, a rapid POC antigen test had $\geq 72 \backslash \%$ sensitivity and $\geq 99 \backslash \%$ specificity vs. RT-PCR. Ann. Intern. Med. 2021; 174: JC83.

[20] Isoldi S. et al. The comprehensive clinic, laboratory, and instrumental evaluation of children with COVID-19: A 6months prospective study. J. Med. Virol. 2021; 93: 3122-3132.

[21] Y\ilmaz, K. \& \cSen, V. Is vitamin D deficiency a risk factor for COVID-19 in children? Pediatr. Pulmonol. 2020; 55: 3595-3601.

[22] Bayramo\uglu, E. et al. The association between vitamin D levels and the clinical severity and inflammation markers in pediatric COVID-19 patients: single-center experience from a pandemic hospital. Eur. J. Pediatr. 2021; $1-7$.

[23] Alpcan A, Tursun S, Kandur Y. Vitamin D levels in children with COVID-19: a report from Turkey. Epidemiol. |\& Infect. 2021; 149.

[24] Söbü E, Karaaslan A, Çetin C, Ak\in, Y. Vitamin D levels of COVID-19 positive sypmtomatic pediatric cases. 2021.

[25] Katz J, Yue S, Xue W. Increased risk for COVID-19 in patients with vitamin D deficiency. Nutrition. 2021; 84, 111106.

[26] Mok CK. et al. Calcitriol, the active form of vitamin D, is a promising candidate for COVID-19 prophylaxis. BioRxiv. 2020.

[27] Biesalski, H. K. Vitamin D deficiency and co-morbidities in COVID-19 patients--A fatal relationship? Nfs J. 2020; 20: 10 .

[28] Jolliffe DA. et al. Vitamin D supplementation to prevent acute respiratory infections: a systematic review and meta-analysis of aggregate data from randomised controlled trials. Lancet Diabetes |\& Endocrinol. 2021.

[29] Holick MF. Sunlight and vitamin D for bone health and prevention of autoimmune diseases, cancers, and cardiovascular disease. Am. J. Clin. Nutr. 2004; 80: 1678S--1688S.

[30] Asyary A, Veruswati M. Sunlight exposure increased Covid-19 recovery rates: A study in the central pandemic area of Indonesia. Sci. Total Environ. 2020; 729: 139016.

[31] Taha, R. et al. The Relationship Between Vitamin D and Infections Including COVID-19: Any Hopes? Int. J. Gen. Med. 2021; 14: 3849.

[32] Holick MF. et al. Evaluation, treatment, and prevention of vitamin D deficiency: an Endocrine Society clinical practice guideline. J. Clin. Endocrinol. |\& Metab. 2011; 96: 1911-1930. 
[33] Zhu, J. \& DeLuca, H. F. Vitamin D 25-hydroxylase--four decades of searching, are we there yet? Arch. Biochem. Biophys. 2012; 523: 30-36.

[34] Zhu JG, Ochalek JT, Kaufmann M, Jones G, DeLuca HF. CYP2R1 is a major, but not exclusive, contributor to 25hydroxyvitamin D production in vivo. Proc. Natl. Acad. Sci. 2013; 110: 15650-15655.

[35] Keane JT, Elangovan H, Stokes RA, Gunton JE. Vitamin D and the liver-correlation or cause? Nutrients. 2018; 10: 496.

[36] Panfili FM. et al. Possible role of vitamin D in Covid-19 infection in pediatric population. J. Endocrinol. Invest. 2021; 44: 27-35.

[37] Kalia V, Studzinski GP, Sarkar S. Role of vitamin D in regulating COVID-19 severity-An immunological perspective. J. Leukoc. Biol. 2021.

[38] Zdrenghea, M. T. et al. Vitamin D modulation of innate immune responses to respiratory viral infections. Rev. Med. Virol. 2017; 27: e1909.

[39] Strollo R, Pozzilli P. DPP4 inhibition: preventing SARS-CoV-2 infection and/or progression of COVID-19? Diabetes. Metab. Res. Rev. 2020; 36: e3330.

[40] Zheng M. et al. Functional exhaustion of antiviral lymphocytes in COVID-19 patients. Cell. |\& Mol. Immunol. 2020; 55: 102763.

[41] Liu J. et al. Longitudinal characteristics of lymphocyte responses and cytokine profiles in the peripheral blood of SARS-CoV-2 infected patients. EBioMedicine. 2020; 55: 102763. 